\title{
Comparison of Depression among Married and Divorced People in Ethiopia: Secondary Data Analysis
}

\section{Abiyu Ayalew Assefa ${ }^{1}$, Kaleab Tesfaye Tegegne ${ }^{1 *}$, Gashaw Mehiret Wubet $^{2}$, Andualem Zenebe ${ }^{1}$, Eleni Tesfaye Tegegne ${ }^{3}$ and Mekibib Kassa Tessema ${ }^{4}$}

${ }^{1}$ Department of Public Health, Hawassa College of Health Science, Hawassa, Ethiopia

${ }^{2}$ Health Science College, School of Medicine, Debretabor University, Debretabor,

Ethiopia

${ }^{3}$ College of Medicine and Health Science, School of Nursing, University of Gondar,

Gondar

${ }^{4}$ Leishmania Research and Treatment Center, University of Gondar, Gondar, Ethiopia

*Corresponding Author: Kaleab Tesfaye Tegegne, Department of Public Health,

Hawassa College of Health Science, Hawassa, Ethiopia.
Received: June 01, 2021

Published: August 28, 2021

(C) All rights are reserved by Kaleab Tesfaye

Tegegne., et al.

\begin{abstract}
Background: In Ethiopia, mental illness in particular depression is the leading non-communicable disorder in terms of burden. The purpose of this review is to provide latest available comparison of depression among married and divorced people in Ethiopia.

Methods: This is systemic review and meta-analysis by study design. The studies were searched in Google Scholar, Medline and Web of science database and PRISMA guidelines protocol was used to write the systematic review.

Results: Twenty studies were found to be eligible and included in the meta-analysis. The combined effect size of all studies revealed a statistically significant association between marital status and depression (OR $=0.57 ; 95 \% \mathrm{CI}: 0.37-0.86$ ).

Conclusion: We found that divorced people were more likely to have depression than married people. Thus, more attention should be paid to strengthen activities which discourage divorce at community level and integrating mental health counseling into health extension programme is advisable.
\end{abstract}

Keywords: Depression; Ethiopia; Marital Status; Mental Disorder

\section{Introduction}

Depression, a mental illness characterized by low mood (sad mood), is one of the most serious and common mental disorders [1].

At a global level, over 300 million people were estimated to suffer from depression in 2015, which was equivalent to $4.4 \%$ of the world's population [2]. Even though, mental health and wellbeing are specifically addressed under SDG Target 3.4, an estimate of one in 10 people in the world suffer from a mental disorder [3]; which make it a barrier to sustainable development in all regions.
The number of persons with common mental disorders globally is going up, particularly in lower-income countries, because the population is growing and more people are living to the age when depression most commonly occurs. The total estimated number of people living with depression increased by $18.4 \%$ between 2005 and 2015 [4]; this reflects the overall growth of the global population, as well as an increase in the age groups at which depression is more prevalent. Eighteen-country adult based study reported that the life time prevalence of Major Depressive Disorder (MDD) was $14.6 \%$ in 10 high income countries and $11.1 \%$ in 8 low and middle income countries [5]. Depression was also reported as $29.6 \%$ in 
Thai [6], 47.3\% in Kathmandu Valley [7], 45.2\% in Thailand [8]. We also found that depression is highly prevalent in Botswana which accounts $25.3 \%$ among women and $31.4 \%$ among men [9].

In Ethiopia, mental illness in particular depression is the leading non-communicable disorder in terms of burden. Nationwide study using data from the Ethiopian National health survey showed depressive episode of 9.1\% [10] and 4.8\% [11] among women. Moreover, the life time prevalence of depression in general population was reported $2.2 \%$ [12].

Depression prevents people from reaching their full potential, impairs human capital, and is associated with premature mortality from suicide and other illnesses and It represents a barrier to sustainable development in all regions $[13,14]$.

Depressive disorders led to a global total of over 50 million Years Lived with Disability (YLD) in 2015 and up to $15 \%$ of individuals with severe depression (about 1 in 7) will die from suicide unless they are properly identified, treated and followed up [1,2].

Although depression can and does affect people of all ages, from all walks of life, the risk of becoming depressed is increased by chemical changes in the brain, poverty, older age, lower educational level, unemployment, life events such as the death of a loved one or a relationship break-up (divorce), lack of adequate support, physical illness and problems caused by alcohol and drug use $[1,10,15]$.

Providing reliable and up-to-date evidence on depression among married and divorced adults especially for country having larger populations like Ethiopia is a key ingredient of effective health policy, planning, evaluation and intervention concerning depression. Additionally, it is useful to indicate that whether there is difference in depression among married and divorced adults and if there is difference which group is at risk for depression so that clinician can use this information for patient management. Therefore, this systemic review and meta-analysis was aimed at providing latest available comparison of depression among married and divorced people in Ethiopia.

\section{Materials and Methods}

Study design and searching strategy

This is systemic review and meta-analysis by study design. The PRISMA guidelines protocol was used to write the systematic review [16]. The studies were searched in Google Scholar, Medline and Web of science database by using the following terms: "depression", "determinants of depression" and Ethiopia. The data used for this review were extracted from articles published between 2012 and 2019 [10,17-35].

\section{Population and sample}

The study population was all people having age of $>=18$. Intervention/exposure is married while comparison is divorced and the outcome was depression.

Inclusion criteria

Studies were included in the review if; (1) the study was an observational study (cross-sectional study, case-control study, or cohort study), (2) the outcome of interest was depression, (3) the study reported data on married and divorced with respect to depression, and (4) studies are published between 2010 and 2020.

\section{Exclusion criteria}

Studies conducted abroad Ethiopia and studies identified depression without tool was excluded.

\section{Measures}

Studies included in this review use different tool to measure depression but all of them dichotomized depression as "yes" if there is depression and "No" if there is no depression. Married and divorced were considered to be indicators of marital status.

\section{Data extraction and synthesis}

Data extraction was made by using data extraction form. We developed the data extraction form that meet the objective of this study. It included year of publication, the name of an author, study design, and number of depression among married and divorced.

\section{Quality assessment}

The assessment of included studies were done using checklist developed by Joanna Briggs Institute which consists of eight points to screen article and all studies have acceptable quality [36].

\section{Statistical analysis}

We used Review Manager Version 5.3 and Meta essential software to calculate combined effect size and associated 95\% confidence intervals so as to compare depression among married and divorced population.

We determined combined effect size across the studies using Mantel-Haenszel (MH) statistic (random effect model) and moderator analysis by taking prevalence of depression as fixed factor. The 
$I^{2}$ statistic was used to assess variation across studies and above $50 \%$ was considered as significant [37]. Finally, Egger's and Begg's tests were used to assess for publication bias [38].

\section{Results}

Search outcomes

The electronic searching of records results in 351 articles. Among 351 research articles, 200 were excluded due to duplication and our inclusion criteria and 103 articles were excluded because title and abstract did not fit our inclusion criteria. 48 of full-text articles assessed for eligibility and 26 of full-text articles excluded, with reasons of not presenting data on depression and marital status.

Finally, twenty two research articles were included to estimate pooled odd ratio of depression among married and divorced adults in Ethiopia (Figure 1).

Study characteristics

The sample size of included studies ranges from 4925 [10] to 264 [25]. All included studies were cross-sectional surveys and done in different regions of Ethiopia. Four studies from Oromia [24,30,32,35], four studies from Amhara region [17,20,22,32], four studies from Addis Ababa [25,27-29], four studies from SNNP
$[18,21,26,34]$, one study from Harari [33], One study from Tigray [31], One study is national from Ethiopia [10] (Table 1).

\section{.}




\section{Comparison of depression}

In this meta-analysis, we examined the association between marital status and depression by using 20 studies.

As we have seen from the frost plot, there are 9731 respondents out of which 3280 of them reported having depression and 8576 of them are married while 1155 are divorced.
The combined effect size of all studies revealed a statistically significant association between marital status and depression. Accordingly, married people were less by $43 \%$ risk of developing depression compared to divorced people (OR $=0.57 ; 95 \% \mathrm{CI}: 0.37$ $-0.86)$.

There is a significant heterogeneity across the studies included $\left(\mathrm{I}^{2}=85 \%\right)$ (Figure 2).

\begin{tabular}{|c|c|c|c|c|c|c|c|c|c|c|}
\hline Study or Subgroup & $\begin{array}{l}\text { marrie } \\
\text { Events }\end{array}$ & married & \multicolumn{2}{|c|}{ divorced } & Weight & Odds Ratio & & \multicolumn{3}{|c|}{ Odds Ratio } \\
\hline Abadiga.2019 & 51 & 132 & 22 & 49 & $5.5 \%$ & $0.77[0.40,1.50]$ & & $\rightarrow$ & & \\
\hline Amha et al.2020 & 175 & 544 & 56 & 96 & $6.0 \%$ & $0.34[0.22,0.53]$ & & $\rightarrow$ & & \\
\hline Azeze et al.2020 & 69 & 264 & 14 & 41 & $5.4 \%$ & $0.68[0.34,1.38]$ & & & & \\
\hline Berhe et al.2013 & 47 & 129 & 23 & 37 & $5.3 \%$ & $0.35[0.16,0.74]$ & & $\rightarrow$ & & \\
\hline Dorsisa et al.2020 & 28 & 125 & 28 & 74 & $5.6 \%$ & $0.47[0.25,0.89]$ & & & & \\
\hline Duko B.et al.2019 & 150 & 188 & 32 & 55 & $5.6 \%$ & $2.84[1.49,5.40]$ & & & $\rightarrow$ & \\
\hline Duko et al.2018 & 88 & 178 & 22 & 45 & $5.5 \%$ & $1.02[0.53,1.97]$ & & & 一 & \\
\hline Edmealem et al.2020 & 7 & 241 & 1 & 22 & $2.4 \%$ & $0.63[0.07,5.35]$ & & & & \\
\hline Ejigu et al. 2020 & 2 & 8 & 33 & 66 & $3.2 \%$ & $0.33[0.06,1.77]$ & & & & \\
\hline Fanta et al.2020 & 22 & 150 & 15 & 44 & $5.3 \%$ & $0.33[0.15,0.72]$ & & & & \\
\hline Feyera et al. 2015 & 146 & 462 & 50 & 100 & $6.0 \%$ & $0.46[0.30,0.72]$ & & & & \\
\hline Habtewold et al.2016 & 72 & 183 & 16 & 24 & $4.9 \%$ & $0.32[0.13,0.80]$ & & & & \\
\hline Hailemariam et al. 2012 & 302 & 3351 & 34 & 234 & $6.1 \%$ & $0.58[0.40,0.85]$ & & & & \\
\hline Minichil et al.2019 & 1251 & 1365 & 72 & 103 & $5.9 \%$ & $4.72[2.97,7.50]$ & & & $\rightarrow$ & \\
\hline Mossie.et al.2016 & 84 & 312 & 9 & 16 & $4.6 \%$ & $0.29[0.10,0.79]$ & & & & \\
\hline Reta et al.2019 & 51 & 115 & 7 & 12 & $4.2 \%$ & $0.57[0.17,1.90]$ & & & & \\
\hline Seid et al. 2020 & 9 & 176 & 8 & 60 & $4.7 \%$ & $0.35[0.13,0.95]$ & & & & \\
\hline Tilahun et al.2018 & 168 & 314 & 35 & 42 & $5.1 \%$ & $0.23[0.10,0.53]$ & & & & \\
\hline Tilahune et al.2016 & 44 & 173 & 4 & 16 & $4.2 \%$ & $1.02[0.31,3.34]$ & & & & \\
\hline Yeshaw et al. 2017 & 24 & 166 & 9 & 19 & $4.7 \%$ & $0.19[0.07,0.51]$ & & & & \\
\hline $\operatorname{Total}(95 \% \mathrm{Cl})$ & & 8576 & & 1155 & $100.0 \%$ & $0.57[0.37,0.86]$ & & & & \\
\hline Total events & 2790 & & 490 & & & & & & & \\
\hline $\begin{array}{l}\text { Heterogeneity. Tau }{ }^{2}=0.7 \\
\text { Test for overall effect } Z=\end{array}$ & $\begin{array}{l}1 ; C h i^{2}=1 \\
2.66(P=0\end{array}$ & $\begin{array}{l}29.81 \\
0.008)\end{array}$ & $d f=19(F$ & $0<0.00$ & 0001): & $\%$ & 0.01 & 0.1 married $^{1}$ & divorced & $100^{\circ}$ \\
\hline
\end{tabular}

Figure 2: Forest plot of the pooled effect size of depression among married and divorced people in Ethiopia, 2021.

\section{Source of heterogeneity}

To identify the possible source of heterogeneity, we undertake moderator analysis by taking prevalence of depression as a moderator by using fixed effect model. Accordingly, the moderator analysis indicated that as prevalence of depression increase the effect size of depression also increase $(B=0.02$, P-value $<0.001)$ (Figure 3).

\section{Reporting bias}

For this analysis, we also assessed publication bias using Begg's and Egger's tests, the result of the test statistics indicated that there was no possible presence of statistically significant publication bias $(\mathrm{p}=0.795)$ and $(\mathrm{p}=0.328)$ respectively (Figure 4$)$. 


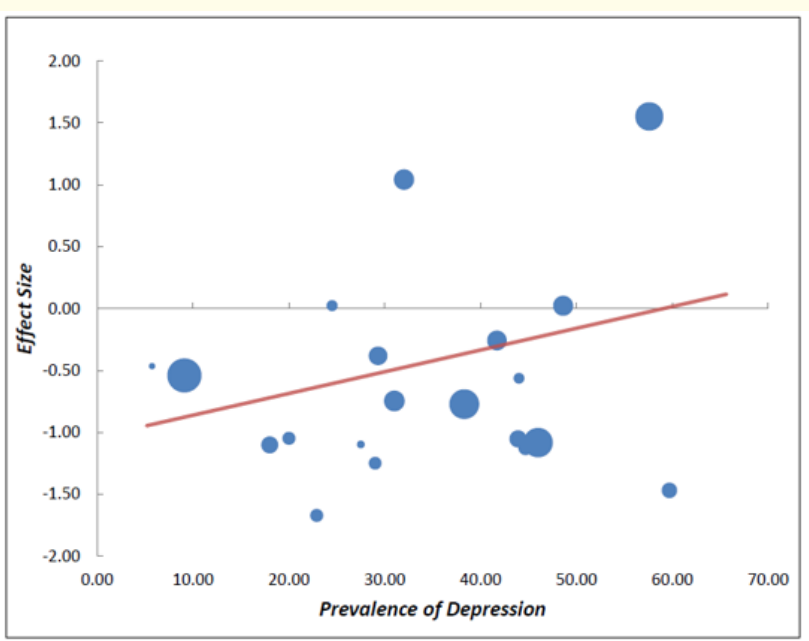

Figure 3: Moderator analysis of prevalence of depression and effect size of depression in Ethiopia, 2021.

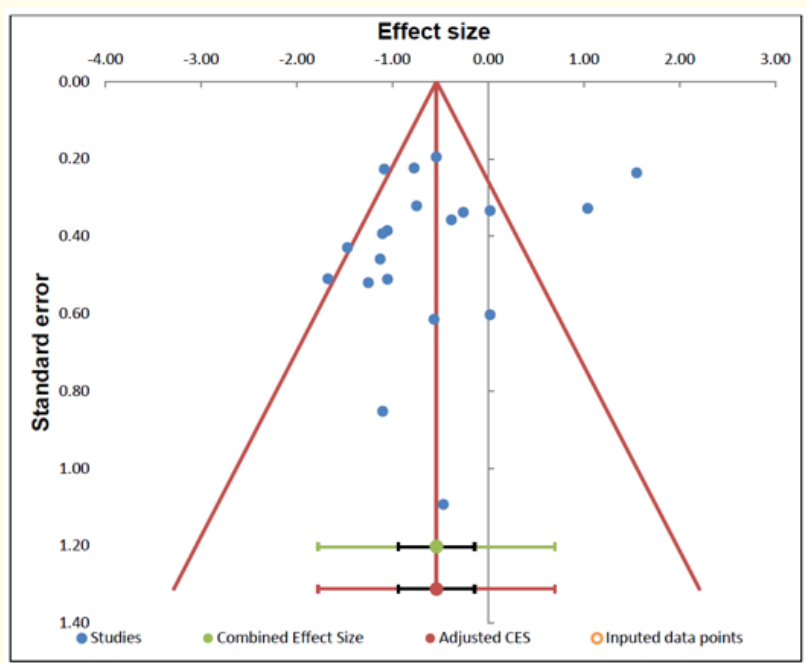

Figure 4: Funnel plot of the comparison between depression and marital status in Ethiopia, 2021.

\section{Discussion}

This systemic review and meta-analysis was done to compare depression among married and divorced peoples in Ethiopia.

The finding of our study suggests that marital status has significant association with depression. Thus, married people have lower chance of depression compared to divorced people.

When compared to the available evidences, our finding was comparable with other studies carried out in different parts of the world like: in India [39], Sri Lanka [40,41], Botswana [9] and South Africa [42] that indicated, being married is a protective factor of depression. Similarly, the finding is in line with previous studies conducted in Ethiopia $[17,19,27,34,43,44]$ that showed being divorced was positively associated with depression.

Potential explanations for this finding might be attributed to a difference in stress coping mechanism between married and divorced people as well as perceived loneliness sensation and loss of social support in divorced people unlike married people who have the opportunity to find emotional support and intimacy in one's partner.

However, our finding is inconsistent with studies done in India [45] and Uganda [46] that showed marital status were not found to be statistically significantly associated with depression. Other study on depression and ART initiation among HIV serodiscordant couples in Kenya and Uganda also reports marital status has no association with probable depression [47]. Additionally, our finding is not comparable studies conducted in several parts of Ethiopia $[18,25,26,31,32]$ which suggest that marital status were not found to be predictors of depression.

The possible reason for this discrepancy might be difference in study population, sample size, study setting, prevalence of depression and lifestyle factors such as sociocultural characteristics, environmental.

\section{Conclusion}

We found that divorced people were more likely to have depression than married people. So psychological support by mental health worker is needed for divorced people so as to decrease the occurrence of depression. More attention should be paid to strengthen activities which discourage divorce at community level and integrating mental health counseling into health extension programme is advisable.

\section{Data Sharing Statement}

All data belong to this study are contained and presented in this document. 


\section{Funding Support}

The authors have not declared a specific grant for this research from any funding agency in the public, commercial or not-for-profit sectors.

\section{Disclosure}

The authors declare that they have no conflicts of interests.

\section{Authors' Contribution}

All authors (AAA, KTT, GMW, ETT, MKT and AZ) made substantial contributions to conception and design, acquisition of data, or analysis and interpretation of data. All authors (AAA, KTT, GMW, ETT, MKT and AZ) read and agreed to submit to the current journal.

\section{Acknowledgments}

We would like to express our deepest gratitude to all the primary authors of the included articles. Finally, we would like to acknowledge Ashley Pfister for screening abstracts and making the PRISMA diagram.

\section{Bibliography}

1. FMOH. "Non-communicable disease prevention and control; learning module for level_III health extension program in Ethiopia" (2014).

2. Depression and Other Common Mental Disorders: Global Health Estimates. Geneva: World Health Organization (2017).

3. WHO. Health in 2015: from MDGs, Millennium Development Goals to SDGs, Sustainable Development Goals (2015).

4. "Global, regional, and national incidence, prevalence, and years lived with disability for 310 diseases and injuries, 1990 - 2015: a systematic analysis for the Global Burden of Disease Study 2015". Lancet 388 (2017): 1545-1602.

5. Bromet E., et al. "Cross-national epidemiology of DSM-IV major depressive episode". BMC Medicine 9.1 (2011): 90.

6. Maneeton B., et al. "Prevalence of Depression and its Correlations: a Cross-sectional Study in Thai Cancer Patients". Asian Pacific Journal of Cancer Prevention 13 (2012): 2039-2043.

7. Kafle B., et al. "Prevalence of Depression among elderly living in old age homes of Kathmandu Valley and its association with Sociodemographic variants". Journal of Psychiatrists' Association of Nepal 4 (2017): 2-7.
8. Louthrenoo BMNMW. "Prevalence and predictors of depression in patients with systemic lupus erythematosus: a crosssectional study". Neuropsychiatric Disease and Treatment 9 (2013):799-804.

9. Gupta R., et al. "Depression and HIV in Botswana: A Population-Based Study on Gender-Specific Socioeconomic and Behavioral Cor- relates". PLoS One 5.12 (2010).

10. Hailemariam S., et al. "The prevalence of depression and associated factors in Ethiopia: findings from the National Health Survey". International Journal of Mental Health Systems 6.23 (2012): 1-11.

11. Deyessa N., et al. "Clinical Practice and Epidemiology Intimate partner violence and depression among women in rural Ethiopia: a cross-sectional study". Clinical Practice and Epidemiology in Mental Health (2009): 10.

12. Fekadu A., et al. "Utility of the concept of minor depressive disorder: Evidence from a large rural community sample in a developing country setting". Journal of Affective Disorders 104 (2007): 111-118.

13. Patel V., et al. "Addressing the burden of mental, neurological, and substance use disorders: key messages from Disease Control Priorities, 3rd edition". Lancet 6736.15 (2015): 1-14.

14. Lund C., et al. "Goals: a systematic review of reviews Review Social determinants of mental disorders and the Sustainable Development Goals: a systematic review of reviews". The Lancet Psychiatry 5.4 (2018): 357-369.

15. Folb N., et al. "Socioeconomic predictors and consequences of depression among primary care attenders with non-communicable diseases in the Western Cape, South Africa: cohort study within a randomised trial". BMC Public Health 15 (2015): 1194.

16. Moher D., et al. "Preferred Reporting Items for Systematic Reviews and Meta-Analyses: The PRISMA Statement". PLOS Medicine 6.6 (2009): 1-2.

17. AmhaH.,etal. "Depression and associated factors among old age population in Dega damot". The Journal of Affective Disorders Reports 2 (2020): 100034.

18. Psychiatry G., et al. "Prevalence and associated factors of depression among patients with HIV / AIDS in Hawassa, Ethiopia, crosssectional study". Annals of General Psychiatry 45 (2018): 4-9. 
19. Feyera F., et al. "Prevalence of depression and associated factors among Somali refugee at melkadida camp, southeast Ethiopia : a cross-sectional study". BMC Psychiatry 171 (2015): $1-7$.

20. Seada Seid and Oumer Abdu MMKST. "Prevalence of depression and associated factors among HIV / AIDS patients attending antiretroviral therapy clinic at Dessie referral". International Journal of Mental Health Systems 55 (2020): 1-8.

21. Duko B., et al. "Depression, Anxiety and Their Correlates Among Patients With HIV in South Ethiopia: An InstitutionBased Cross-Sectional Study". Front Psychiatry 10 (2019): 1-7.

22. Edmealem A and Olis CS. "Factors Associated with Anxiety and Depression among Diabetes, Hypertension, and Heart Failure Patients at Dessie Referral Hospital, Northeast Ethiopia". Behavioral Neurology (2020): 1-10.

23. Reta Y and G/Egziabher RGMBBKKWY. "Depressive disorder and its associated factors among prisoners in Debre Berhan Town, North Showa, Ethiopia (2019).

24. Yeshaw Y and Mossie A. "Depression, anxiety, stress, and their associated factors among Jimma University staff, Jimma, Southwest Ethiopia, 2016: a cross-sectional study". Neuropsychiatric Disease and Treatment 13 (2017): 2803-2812.

25. Habtewold TD., et al. "Sociodemographic, clinical, and psychosocial factors associated with depression among type 2 diabetic out- patients in Black Lion General Specialized Hospital, Addis Ababa, Ethiopia: a cross-sectional study". BMC Psychiatry 103 (2016): 1-8.

26. Azeze GA., et al. "Factors Associated with Untreated Depression Among Type 2 Diabetic Patients at Halaba Kulito Hospital, South Ethiopia: A Cross-Sectional Study". Diabetes, Metabolic Syndrome and Obesity: Targets and Therapy 13 (2020): 2189-2198.

27. Ejigu AK., et al. "Depression, anxiety and associated factors among housemaids working in Addis Ababa Ethiopia". BMC Psychiatry 20.231 (2020): 1-11.

28. Minichil W., et al. "Depression and associated factors among primary caregivers of children and adolescents with mental illness in Addis". BMC Psychiatry 249 (2019): 1-9.

29. Fanta T., et al. "The prevalence and associated factors of depression among patients with schizophrenia in Addis Ababa, Ethiopia, cross-sectional study". BMC Psychiatry 3 (2020): 1-7.
30. Abadiga M. "Depression and its associated factors among HIV / AIDS patients attending ART clinics at Gimbi General hospital, West Ethiopia”. BMC Research Notes 527 (2019): 1-8.

31. Haftu Berhe AB. "Prevalence Of Depression And Associated Factors Among People Living With Hiv/Aids In Tigray, North Ethiopia: A Cross Sectional Hospital Based Study". International Journal of Pharmaceutical Sciences and Research 4.2 (2013): 765-775.

32. Dorsisa B., et al. "Prevalence and Factors Associated with Depression among HIV / AIDS-Infected Patients Attending ART Clinic at Jimma University Medical Center, Jimma, Southwest Ethiopia". Psychiatry Journal (2020): 1-9.

33. Tilahun H., et al. "Depression and Associated Factors among Adult Inpatients at Public Hospitals of Harari Regional State, Eastern Ethiopia". Psychiatry Journal (20118): 1-6.

34. Tamiru Asres BT Gezahegn BNM eyerusalem. "Prevalence of unrecognized depression and associated factors among patients attending medical outpatient department in Adare Hospital". American Journal of Psychiatry 12 (2016): 2723-2729.

35. Mossie A., et al. "Prevalence and Severity of Depression and Its Association with Substance Use in Jimma Town, Southwest Ethiopia". Depression Research and Treatment (2016): 1-7.

36. Institute JB. Critical Appraisal Checklist for Analytical Cross Sectional Studies (2017).

37. Higgins JPT., et al. "Measuring Inconsistency in Meta-Analyses Measuring inconsistency in meta-analyses". Education Debates 327 (2003): 557-560.

38. Borenstein M., et al. "A basic introduction to fixed-effect and random-effects models for meta-analysis". Research Synthesis Methods 1 (2010): 97-111.

39. Kulatunga OBWRSSPM. "Prevalence of Unrecognised Depression Among Outpatient Department Attendees of A Rural Hospital in Delhi, India". Journal of Clinical and Diagnostic Research 7.9 (2013): 1921-1925.

40. Arambewela MH., et al. "Prevalence of Depression and Associated Factors among Patients with Type 2 Diabetes Attending the Diabetic Clinic at a Tertiary Care Hospital in Sri Lanka: A Descriptive Study". Psychiatry Journal (2019): 1-8. 
41. Sri Lanka-ye-1-Prevalence and correlates of depression among older urban community-dwelling adults in Sri Lanka - Rajapakshe - 2019 - Psychogeriatrics - Wiley Online Library (2019).

42. Padayachey U., et al. "Depression in older adults : prevalence and risk factors in a primary health care sample Depression in older adults : prevalence and risk factors in a primary health care sample". The South African Family Practice 6190 (2017): 61-66.

43. Hajure M., et al. "Depression, Anxiety and Associated Factors Among Chronic Medical Patients Amid COVID-19 Pandemic in Mettu Karl Referral". Neuropsychiatric Disease and Treatment 16 (2020): 2511-2518.

44. Molla GL., et al. "Depression among Ethiopian Adults: CrossSectional Study". Psychiatry Journal (2016): 14-19.

45. Sengupta $P$ and Benjamin AI. "Prevalence of Depression and Associated Risk Factors among the Elderly in Urban and Rural Field Practice Areas of a Tertiary Care Institution in Ludhiana". Indian Journal of Public Health Research 59.1 (2015): 3-8.

46. Hatcher AM., et al. "Sexual Relationship Power and Depression among HIV- Infected Women in Rural Uganda”. PLoS One 7.12 (2012): 6-12.

47. Velloza J., et al. "Depression and ART initiation among HIV serodiscordant couples in Kenya and Uganda". AIDS and Behavior 21.8 (2018): 2509-2518.

\section{Volume 4 Issue 9 September 2021}

(C) All rights are reserved by Kaleab Tesfaye Tegegne.,

et al. 